\title{
Interval analysis of repetitive denervation potentials of human skeletal muscle
}

\author{
B. CONRAD ${ }^{1}$, F. SINDERMANN, AND V. J. PROCHAZKA \\ From the Department of Neurology and Neurophysiology, \\ University of Ulm, 79 Ulm, West-Germany
}

SUMMARY Interval analyses of denervation potentials (fibrillation-potentials and positive sharp waves) in human skeletal muscles were performed using a DEC PDP-12 computer. Extremely small differences between consecutive intervals (usually less than $1 \%$ ) and slow up and down drifts in the spontaneous discharge frequency were found to be characteristic of repetitive denervation potentials. These two findings are safe criteria for the clinical diagnosis of denervation and they may be superior to the more commonly used criteria based upon the shapes, amplitudes, and durations of the potentials. Physiological data from the literature as well as hypotheses relating to spontaneous activity are reviewed. Generator potentials across the muscle fibre membrane, lowered firing threshold, and constant-sized after-potentials are considered the basic factors relevant to spontaneous and rhythmical firing and to the slow drift observed in the firing frequency.

This study is concerned with the results of a computer analysis of the durations of intervals between repetitively discharging fibrillation potentials and positive sharp waves in the human electromyogram (EMG). A number of criteria serve to identify these potentials: spontaneous discharge, shape, amplitude, and duration of the potential and the discharge frequency (Jasper and Ballem, 1949; Lundervold and Li, 1953; Jarcho, Vera, McCarthy, and Williams, 1958; Buchthal and Rosenfalck, 1966). Regularity of consecutive intervals has been observed (Denny-Brown and Pennybacker, 1938; Kugelberg and Petersen, 1949; Landau, 1951; Jarcho et al., 1958; Buchthal and Rosenfalck, 1966) but has not been considered to be an outstanding feature of human denervation potentials nor has it been expressed in quantitative terms.

For the experienced investigator, difficulty rarely arises in the identification of denervation potentials. In some cases, however, the normal criteria may not suffice. Buchthal and Rosenfalck (1966) found biphasic potentials in undoubtedly normal muscles which could not be differentiated from fibrillation potentials. Moreover, short

1 Present address: Department of Physiology, University of Western Ontario, London, Canada. durations of voluntary action potentials-for example, in facial muscles-as well as the lack of relaxation of the muscle may present serious obstacles to the identification of spontaneous denervation potentials.

In the following paragraphs extreme regularity of consecutive discharge intervals and characteristic slow changes in the discharge frequency will be shown to be additional and safe criteria for the diagnosis of denervation activity in the human electromyogram.

\section{METHODS}

The EMG records from 27 patients were analysed using an automatic unit recognition system. The age of the patients varied between 17 and 64 years. Sixteen patients suffered from traumatic lesions of peripheral nerves, four from polyneuropathy, three from amyotrophic lateral sclerosis, two from poliomyelitis, one from metastatic carcinoma in the brachial plexus, and one from toxic myopathy.

EMGs were recorded with a two-channel Tönnies electromyograph (M46), using a time constant of $2 \mathrm{msec}$ and upper cut-off frequency of $2 \mathrm{KHz}$. Concentric needle electrodes (DISA $9013 \mathrm{Koo31}$ ) were used. Electrical activity was recorded from at least six different sites in a given muscle. Records were 


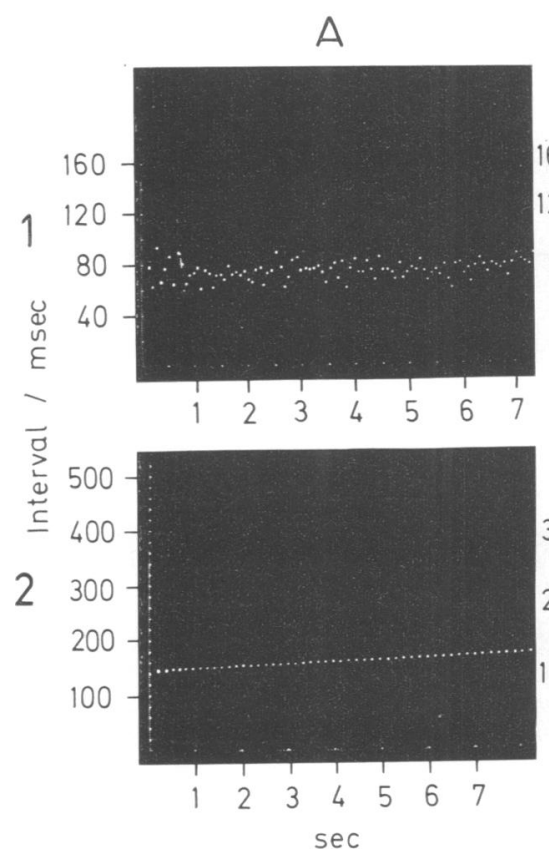

sec
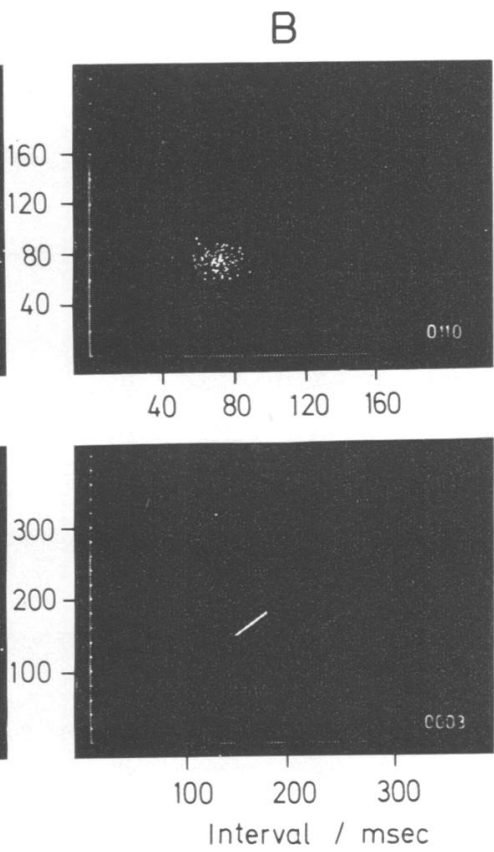

FIG. 1. Interval versus time histograms (left pair) and joint interval histograms (right pair) of voluntary activity of a single motor unit (A) and of a repetitive fibrillation potential $(B)$. Number in lower right corner of joint interval histogram is the APCID (see 'Methods' section). stored on magnetic tape (Sangamo 3000). Records which contained spontaneous denervation potentials were selected for further analysis. The selection was based upon the following criteria: repetitivity (at least 20 consecutive discharges), durations of less than $2 \mathrm{msec}$ in the case of fibrillation potentials, initial positive sharp deflection followed by slow negative after-potential in the case of positive sharp waves. No differentiation was attempted between prolonged insertion activity and true spontaneous activity.

Interval analysis was performed with a medium sized laboratory computer (PDP-12). The analysis is based upon the automatic shape recognition of the potentials. A maximum of four different potentials out of a record containing up to seven potentials are analysed in a single run. Further details of the method are described in a previous paper (Prochazka, Conrad, and Sindermann, 1972). Two different types of interval histograms were available on the computer display: interval versus time histograms and joint interval histograms. In the latter histograms, the coordinates $(\mathrm{x}, \mathrm{y})$ of a given point represent the durations of the consecutive intervals $(n, n+1)$. A numerical value for proportional differences between consecutive interval durations - that is, a measure of the degree of relative irregularity of subsequent intervals, was obtained using the 'average proportional consecutive interval difference' (APCID):

$$
\operatorname{APCID}=\frac{1000}{n} \sum_{i=2}^{i=n} \frac{\left(I_{i}-I_{i-1}\right) /\left(I_{i}+I_{i-1}\right)}{2}
$$

where $I_{i}$ is the duration of the $i$ th interval and $n$ is the total number of intervals $(n+1$ being the total number of spikes).

\section{RESULTS}

CONSECUTIVE DISCHARGE INTERVALS From a total of 242 repetitive denervation potentials (137 fibrillation potentials and 105 positive sharp waves) only 12 fibrillation potentials and seven positive sharp waves showed irregular discharges (APCID greater than 0100 corresponding to an average proportional difference between consecutive intervals of more than $10 \%$ ). The APCID was less than 20 in 122 instances $(89 \%$ of the total) of fibrillation potentials and in 91 instances $(87 \%$ of the total) of positive sharp waves. In 88 instances $(64 \%)$ of fibrillation potentials and in 74 instances $(70.5 \%)$ of positive sharp waves the APCID was less than $10(1 \%$ 

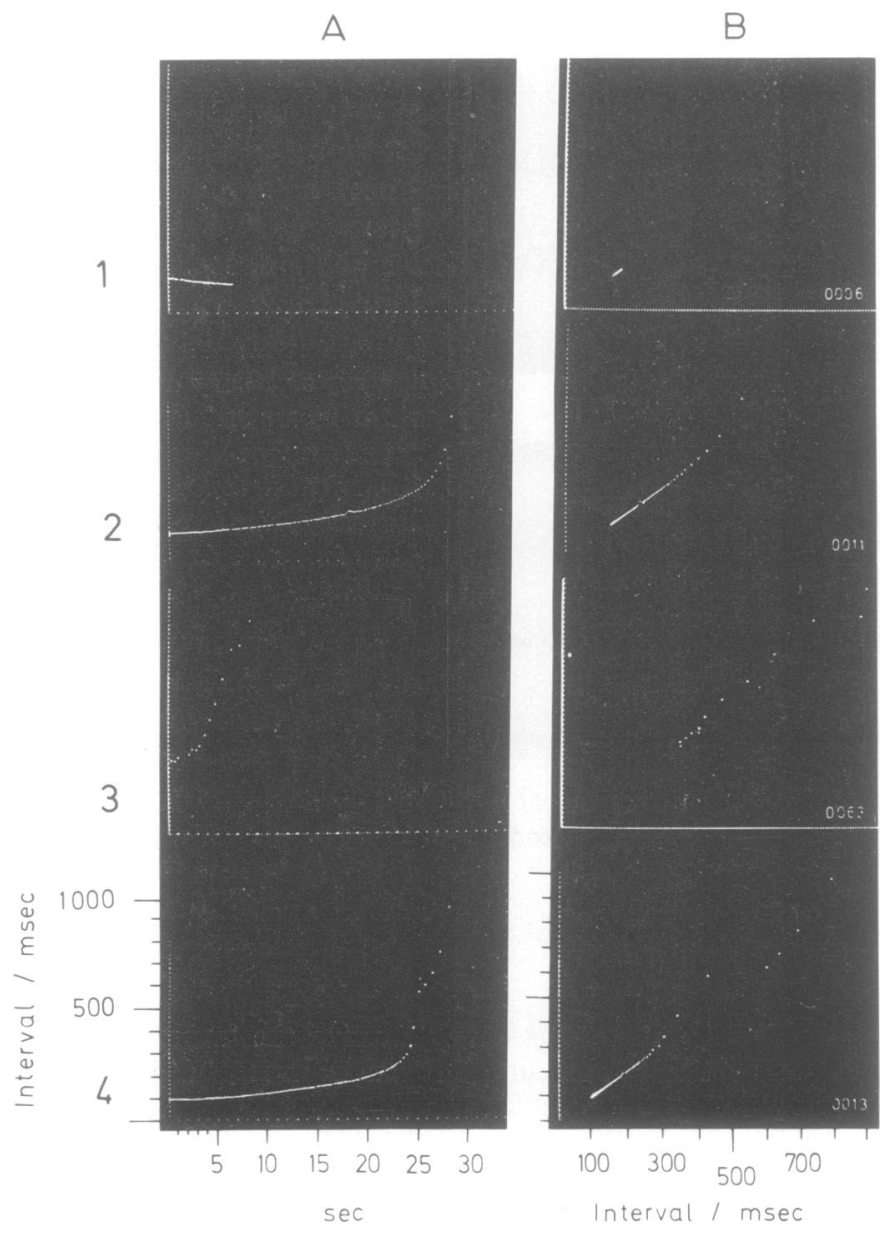

FIG. 2. Interval plots of four different denervation potentials (fibrillations and positive sharp waves) from a single record. (1 to 4). Left column (A): interval versus time histograms. Right column (B): joint interval histograms. Number in lower right corner of joint interval histograms is the APCID.

average proportional difference between consecutive intervals). Even in cases of APCIDs between 20 and 100 many consecutive intervals differed by less than $1 \%$, which is never found in voluntary activity of motor units (Fig. 1A). This extreme regularity is well demonstrated by interval versus time plots and by joint interval histograms (Figs 1 to 3 ). It is often difficult at first glance to identify the consecutive spikes of a given fibrillation potential. Figure 4 shows two records where regular discharge intervals of different denervation potentials might easily be mistaken for irregular discharges if regularity were not expected. True irregularity does occur, but in such cases the trains very rarely exceed 20 discharges. One reason for irregularity is the mutual influence of neighbouring denervation potentials (see last section of results).

SLOW DRIFTS IN DISCHARGE FREQUENCY All denervation potentials which showed regularity of consecutive interval durations also showed drifts in the discharge frequency (Figs 1 to 3 ). Typically the frequency decreased before the fibres ceased to discharge and, not infrequently, the initial discharge frequency also was lower than the average discharge frequency. The drifts in the discharge frequency combined with extremely small differences between consecutive interval durations result in joint interval histograms where the points form a straight line with an approximate slope of $45^{\circ}$ (Figs 1 to 3 ). 


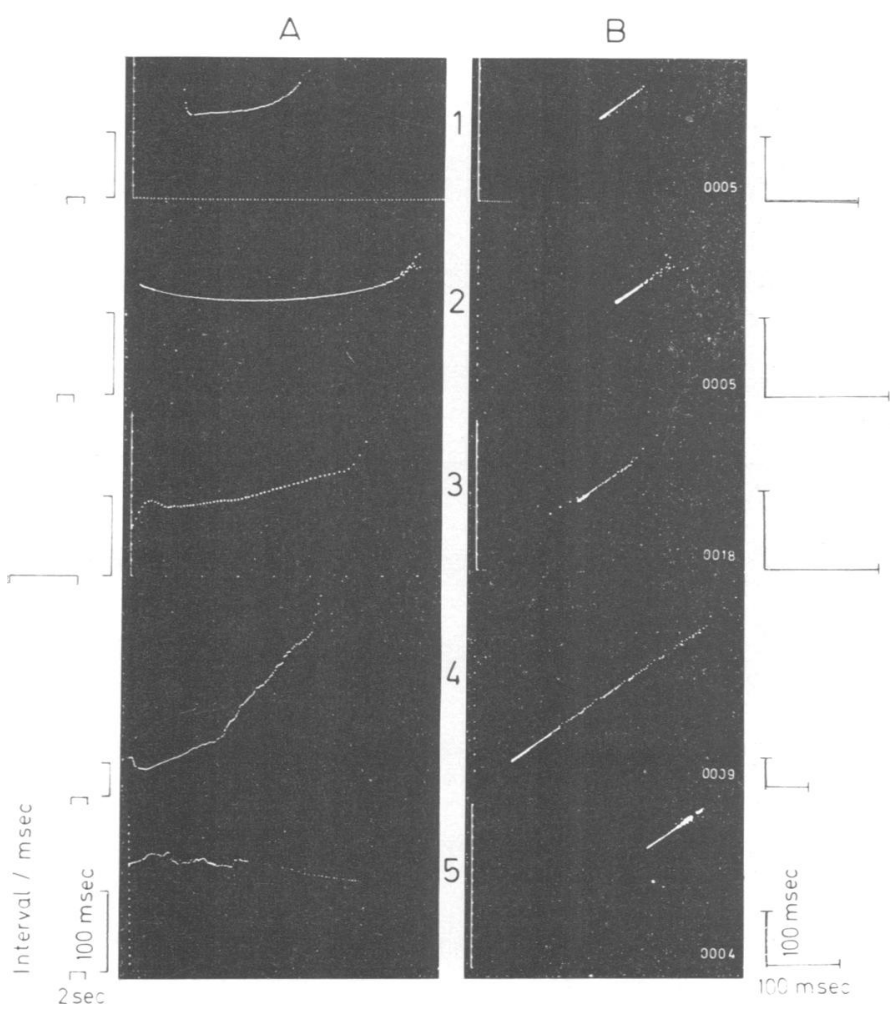

FIG. 3. Interval plots of denervation potentials from five different subjects (1 to 5). Left column (A): interval versus time histograms. Right column (B): joint interval histograms. APCID in lower right corner of joint interval histograms.

DISTRIBUTION OF DISCHARGE INTERVALS For a total of 137 fibrillation potentials the average maximum discharge frequency was $6 \cdot 31 \pm 0 \cdot 54$ spikes $/ \mathrm{sec}(95 \%)$. The average maximum discharge frequency was calculated from the total of 137 individual maximum discharge frequen- cies. For 105 positive sharp waves the average maximum discharge frequency was $7 \cdot 41 \pm 0.44$ spikes $/ \mathrm{sec}(95 \%)$. The standard deviations were 2.6 and 2.7 spikes/sec for fibrillation potentials and positive sharp waves respectively. The maximum individual discharge frequency as



FIG. 4. Two EMG records (A) and (B) from denervation potentials which discharged rhythmically (see arrows) but where rhythmicity is partly obscured by simultaneous recording of different potentials. 


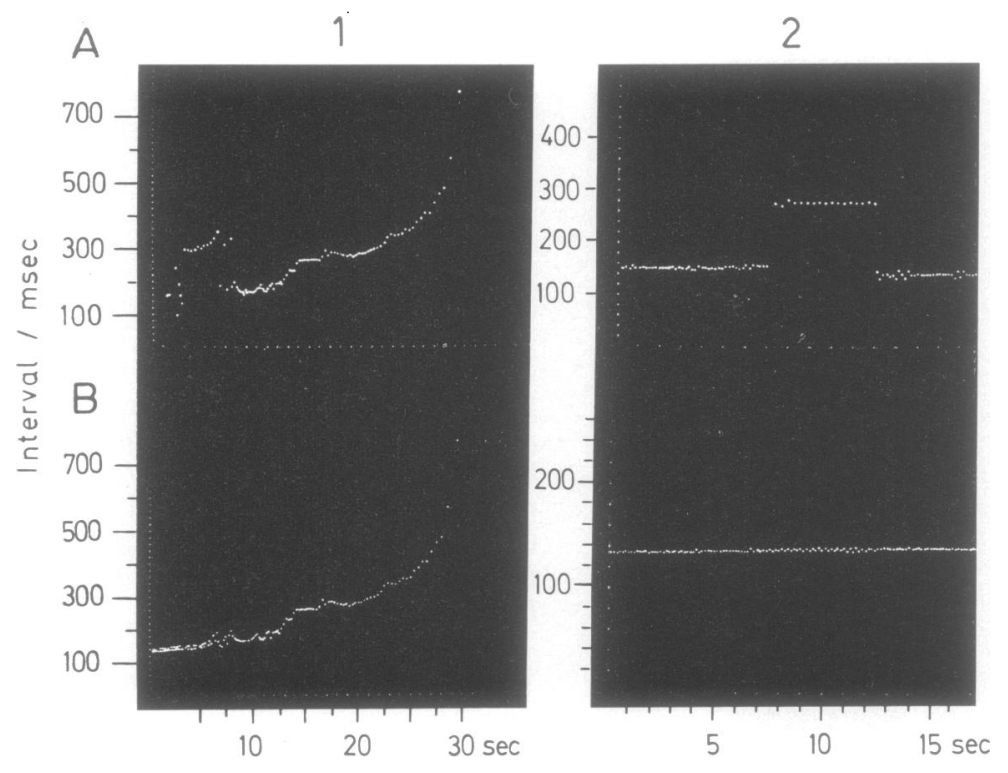

FIG. 5. Interval versus time histograms of neighbouring fibrillation potentials with mutual discharge influences. Left column (1): Fibrillation of one fibre (A) is synchronized with fibrillation of $a$ neighbouring one (B). Right column (2): Fibrillation in upper record (A) is synchronized with every second discharge of fibrillation in lower record (B).

observed in a total of 242 denervation potentials was 14.8 spikes/sec. In a given muscle, simultaneously discharging fibres usually differed in their discharge frequencies.

SYNCHRONIZATION AND OTHER KINDS OF MUTUAL DISCHARGE INFLUENCE Sometimes a fibre suddenly changed its discharge frequency (Figs 5 and 6) evidently influenced by the discharges of a neighbouring fibre. For instance, every second or third discharge of a given fibre was blocked, or even true synchronization of the discharges of neighbouring fibres occurred. Usually, both fibres mutually influenced each other, in that the discharges of the one more or less followed the discharges of the other, while the discharges of the other became more irregular than they were previously. Figure 5 shows how the discharge frequency of a fibrillation potential abruptly decreases and synchronization occurs with a neighbouring fibre (lower trace) which, in turn, shows marked irregularity until the synchronization is achieved. In Fig. 5B the discharge intervals of a fibrillation potential (upper trace) are intermittently increased to twice the discharge intervals of a neighbouring fibre (lower trace), which, during this period of time, shows increased irregularity in its own discharges; the former fibrillation potential synchronized with every second discharge of the latter fibrillation. In Fig. 6 mutual discharge influences are evidenced by $\stackrel{\vec{D}}{\perp}$ the changes in the discharge frequencies of two neighbouring fibres after a third fibre has ceased $\stackrel{\Phi}{\Phi} \vec{\square}$ to discharge.

\section{DISCUSSION}

Repetitive denervation potentials of more than $\bar{\partial}$ 20 consecutive discharges show, in a great majority of cases, extremely low differences between consecutive interval durations. In our material, the mean of these differences was below $1 \%$ of the corresponding interval durations in two-thirds of the instances and exceeded $2 \%$ in only 32 out of 242 different potentials. In spite of this regularity, the discharge frequency never remained constant over longer periods but showed up and down drifts. These two factsregularity of consecutive intervals combined with steady slow changes in the discharge frequency-are well illustrated by the interval versus time histograms and first order joint interval histograms. In the latter type of histogram, the result is approximately a straight line with a slope of $45^{\circ}$-that is, $I_{i} \approx I_{i+1}$. This regularity is never observed in voluntary activity and not even in tetany. In our experience, it usually occurs in myotonia, and it could be 


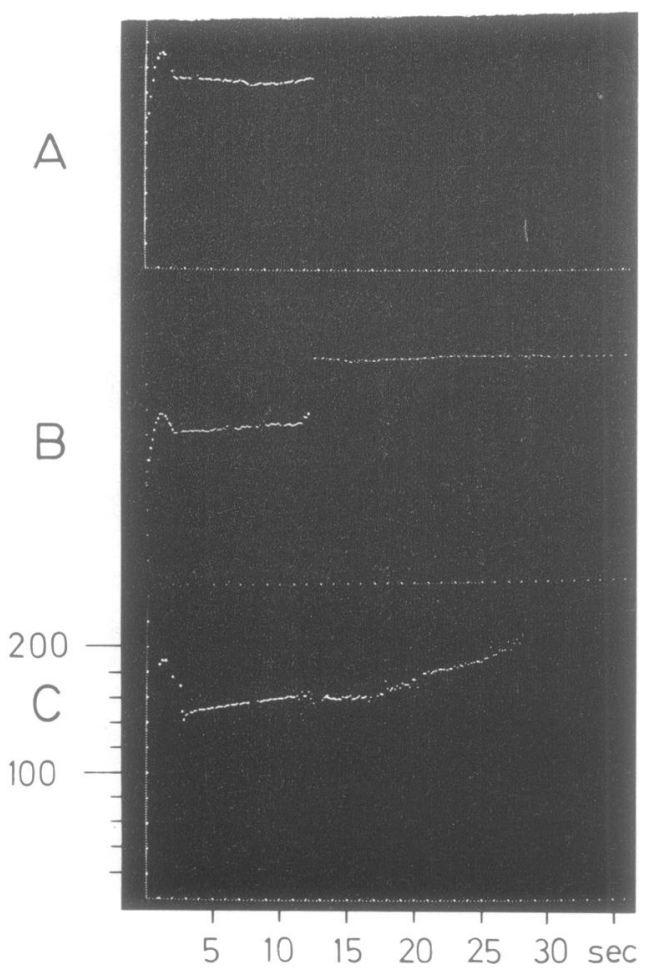

FIG. 6. Interval versus time histograms of three simultaneously discharging fibrillation potentials ((A) to (C)) obtained from a single record. Note sudden changes in discharge intervals in (B) and in discharge regularity in (C) after fibrillation in fibre (A) has ceased.

expected to occur in 'high frequency discharge', though we have not yet investigated this condition. In myotonia and in 'high frequency discharge', however, the maximum discharge frequency is greater than $50 / \mathrm{sec}$, whereas it is usually not higher than $20 / \mathrm{sec}$ in repetitive denervation activity. Landau (1951) has observed a discharge frequency of $100 / \mathrm{sec}$ in denervation potentials, but this is certainly exceptional. In our material, the highest discharge frequency was 15 per second from a total of 242 instances from 24 patients.

Rhythmical discharges of denervation potentials have been reported previously but the regularity has not been considered one of the significant features, nor has the degree of regularity been numerically defined (see above).
There may be several reasons for this. One is that the data might have been expressed as a distribution of intervals durations rather than a sequential plot. In this case, the small differences between subsequent intervals might have been obscured by a drift in the discharge frequency.

Irregular intervals do occur in denervation potentials, even in long trains of discharges. We have only observed them rarely, however, although we might have missed some more when we selected needle positions during EMG recording.

It is not possible to give a meaningful explanation of the basic mechanism of regular spontaneous firing from our data. There are, however, experimental data and adequate hypotheses in the literature which might well explain this extremely regular activity. Nicholls (1956) has shown that the membrane threshold is lowered in denervated muscle fibres; Lüllmann (1960) demonstrated by intracellular recordings that the spontaneous action potential of denervated muscle fibres is followed by marked hyper- and depolarizations. Belmar and Eyzaguirre (1966) proposed the hypothesis that repetitive spontaneous discharges occur during a condition of prolonged depolarization of the membrane potential similar to generator potentials of receptors. Various stimuli (chemical, mechanical, electrical) might give rise to this prolonged depolarization. A generator potential of this kind combined with a low firing threshold and after-potentials of constant size would explain the spontaneity and extreme rhythmicity of the discharges as well as slow trends in the discharge frequency (during changes in the generator potential). In our material, one reason for irregularity of discharge was the synchronization of the discharges of neighbouring fibres, the latter subsequently losing their former regularity. This synchronization may well be due to volume conducted 'cross-talk'.

No differences were found between fibrillation potentials and positive sharp waves with regard to discharge frequency (including slow drifts) and regularity of subsequent intervals. Consequently, the assumed difference between the two sets of denervation potentials (non-injured fibre in the case of fibrillation and injured fibre in the case of positive sharp wave) does not seem to influence the spontaneous discharge rhythmicity. 
It was mentioned in the introduction that identification of spontaneous activity by the shape, the duration, and the voltage of the potentials is sometimes difficult. Additional criteria may be required. Extremely small differences between consecutive intervals and characteristic slow changes in the spontaneous discharge frequency provide such additional criteria.

\section{REFERENCES}

Belmar, J., and Eyzaguirre, C. (1966). Pacemaker site of fibrillation potentials in denervated mammalian muscle. Journal of Neurophysiology, 29, 425-441.

Buchthal, F., and Rosenfalck, P. (1966). Spontaneous electrical activity of human muscle. Electroencephalography and Clinical Neurophysiology, 20, 321-336.

Denny-Brown, D., and Pennybacker, J. B. (1938). Fibrillation and fasciculation in voluntary muscle. Brain, 61, 311334.
Jarcho, L. W., Vera, C. L., McCarthy, C. G., and Williams, P. M. (1958). The form of motor-unit and fibrillation potentials. Electroencephalography and Clinical Neurophysiology, 10, 527-540.

Jasper, H., and Ballem, G. (1949). Unipolar electromyograms of normal and denervated human muscle. Journal of Neurophysiology, 12, 231-244.

Kugelberg, E., and Petersén, I. (1949). 'Insertion activity' in electromyography. Journal of Neurology, Neurosurgery, and Psychiatry, 12, 268-273.

Landau, W. M. (1951). Synchronization of potentials and response to direct current stimulation in denervated mammalian muscle. Electroencephalography and Clinical Neurophysiology, 3, 169-182.

Lüllmann, H. (1960). Über die Ursache spontaner Fibrillationen denervierter Skeletmuskulatur. Klinische Wocherschrift, 38, 1169-1170.

Lundervold, A., and Li, C. L. (1953). Motor units and fibrillation potentials as recorded with different kinds of needle electrodes. Acta Psychiatrica et Neurologica Scandinavica, 28, 201-212.

Nicholls, J. G. (1956). The electrical properties of denervated skeletal muscle. Journal of Physiology, 131, 1-12.

Prochazka, V. J., Conrad, B., and Sindermann, F. (1972). A neuroelectric signal recognition system. Electroencephalography and Clinical Neurophysiology, 32, 95-97. 